\title{
Article
}

\section{"When you haven't got much of a voice": An evaluation of the quality of Independent Mental Health Advocate (IMHA) Services in England}

Newbigging, Karen, Ridley, Julie, Mckeown, Michael, Machin, Karen and Poursanidou, Konstantina

Available at http://clok.uclan.ac.uk/10968/

Newbigging, Karen, Ridley, Julie ORCID: 0000-0002-0879-308X, Mckeown, Michael ORCID: 0000-0003-0235-1923, Machin, Karen and Poursanidou, Konstantina (2015) "When you haven't got much of a voice": An evaluation of the quality of Independent Mental Health Advocate (IMHA) Services in England. Health and Social Care in the Community, 23 (3). pp. 313-324. ISSN 0966-0410

It is advisable to refer to the publisher's version if you intend to cite from the work. http://dx.doi.org/10.1111/hsc.12153

For more information about UCLan's research in this area go to http://www.uclan.ac.uk/researchgroups/ and search for < name of research Group>.

For information about Research generally at UCLan please go to http://www.uclan.ac.uk/research/

All outputs in CLoK are protected by Intellectual Property Rights law, including Copyright law. Copyright, IPR and Moral Rights for the works on this site are retained by the individual authors and/or other copyright owners. Terms and conditions for use of this material are defined in the policies page. 


\title{
'When you haven't got much of a voice': an evaluation of the quality of Independent Mental Health Advocate (IMHA) services in England
}

\author{
Karen Newbigging MA (Hons) MSc DipPsych PhD C Psychol AFBPS FRSPH ${ }^{1}$, Julie Ridley BA (Hons) PhD ${ }^{2}$, Mick \\ McKeown BA (Hons) RGN RMN PhD ${ }^{3}$, Karen Machin BSc (Hons) MPhil ${ }^{3}$ and Konstantina Poursanidou BA (Hons) MEd PhD ${ }^{4}$ \\ ${ }^{1}$ Health Services Management Centre, University of Birmingham, Birmingham, UK, ${ }^{2}$ School of Social Work, University \\ of Central Lancashire, Preston, UK, ${ }^{3}$ School of Health, University of Central Lancashire, Preston, UK and ${ }^{4}$ Centre for \\ Women's Mental Health, University of Manchester, Manchester, UK
}

Accepted for publication 11 August 2014

\author{
Correspondence \\ Karen Newbigging \\ Health Services Management \\ Centre (HSMC) \\ University of Birmingham \\ Park House, 40 Edgbaston \\ Park Road \\ Birmingham B15 2RT, UK \\ E-mail: k.v.newbigging@bham.ac.uk
}

\section{What is known about this topic}

- Not all psychiatric wards in England have access to Independent Mental Health Advocate (IMHA) services, and only a minority of those eligible access support from an IMHA in the tribunal process.

- Inadequate understanding of entitlement to advocacy by professionals, who have a duty to promote access, has been implicated in limited uptake of IMHA.

- Gaps in mental health advocacy provision for those with specific needs reflect inadequate commissioning and models of advocacy provision.

\section{What this paper adds}

- A rigorous in-depth evaluation of multiple perspectives on the provision and experience of statutory mental health advocacy.

- Identification of three factors influencing the quality of IMHA services: effectiveness of commissioning, provision of IMHA services, and understanding and disposition of mental health services to advocacy.

- IMHAs can enhance personal agency in situations where freedoms and control are constrained.

\begin{abstract}
Advocacy serves to promote the voice of service users, represent their interests and enable participation in decision-making. Given the context of increasing numbers of people detained under the Mental Health Act and heightened awareness of the potential for neglect and abuse in human services, statutory advocacy is an important safeguard supporting human rights and democratising the social relationships of care. This article reports findings from a national review of Independent Mental Health Advocate (IMHA) provision in England. A qualitative study used a two-stage design to define quality and assess the experience and impact of IMHA provision in eight study sites. A sample of 289 participants -75 focus group participants and 214 individuals interviewed - including 90 people eligible for IMHA services, as well as advocates, a range of hospital and community-based mental health professionals, and commissioners. The research team included people with experience of compulsion. Findings indicate that the experience of compulsion can be profoundly disempowering, confirming the need for IMHA. However, access was highly variable and more problematic for people with specific needs relating to ethnicity, age and disability. Uptake of IMHA services was influenced by available resources, attitude and understanding of mental health professionals, as well as the organisation of IMHA provision. Access could be improved through a system of opt-out as opposed to opt-in. Service user satisfaction was most frequently reported in terms of positive experiences of the process of advocacy rather than tangible impacts on care and treatment under the Mental Health Act. IMHA services have the potential to significantly shift the dynamic so that service users have more of a voice in their care and treatment. However, a shift is needed from a narrow conception of statutory advocacy as safeguarding rights to one emphasising self-determination and participation in decisions about care and treatment.
\end{abstract}

Keywords: empowerment, evaluation research, Mental Health Act, mental health advocacy, policy research, users' views, rights 


\section{Introduction}

In many countries, the law gives the state powers to detain people with mental health problems in hospital against their will. In England, this is done under the 1983 Mental Health Act (MH Act HMSO, 1983) and is commonly referred to as detention or sectioning. This Act permits people to be treated without their consent and the term compulsion is used to refer to both treatment and/or detention under the Act. The 2007 Mental Health Act extended the powers of compulsion, requiring people on Community Treatment Orders (CTO), to comply with treatment. The increasing numbers of people subject to the Mental Health Act in England is rising, although reasons for this are not well understood (House of Commons Select Committee 2013). Mental health services face an inherent tension in care delivery, with evidence of cultures where control and containment are prioritised over treatment and support evident (Care Quality Commission (CQC) 2012). Amidst service failures and an alleged compassion deficit, the necessity of fully hearing the voice of service users is paramount (Department of Health 2012, Randall \& McKeown 2014). Reflecting a foundational principle of autonomy, the World Health Organisation (WHO 2003) promotes advocacy as a mechanism to ensure rights and reduce discrimination against people with mental health problems. People subject to compulsory treatment are particularly vulnerable, and an underpinning principle of the $\mathrm{MH}$ Act is the active involvement of detained patients in their own care (Department of Health 2008). Independent Mental Health Advocate (IMHA) services were introduced in England under the $2007 \mathrm{MH}$ Act to safeguard rights of eligible patients and promote self-determination. However, although there is a wealth of practice knowledge (NIMHE 2008, NMHDU 2009), there are few evaluative studies to inform commissioning and delivery of high-quality IMHA services. This study was commissioned by the Department of Health (DH) in 2010 to redress this gap.

In reporting our findings, we recognise language describing people who experience mental distress is contested (Wallcraft \& Nettle 2009). We use 'service user' to refer to people using mental health services in general, including those who do not qualify for IMHA services; 'qualifying patient' refers to those entitled to IMHA services - as per the MH Act - and 'advocacy partner' for those using IMHA services.

\section{Background}

The terms 'advocate' and 'advocacy' are widely used and open to interpretation. In the health and social care context, advocacy enables people to have a voice and recognises disparities in power between professionals and service users (Silvera \& Kapasi 2002). Hence, advocacy is understood as a mechanism for promoting self-determination through enhancing participation in decision-making about care. The advocacy role is to represent an individual's concerns (Henderson \& Pochin 2001), 'responding or representing the person's interests as if they were their own' (O'Brien 1987, p. 3). Originating within disability rights and user movements (Barnes 2007), the main purpose of advocacy is empowerment, challenging professional paternalism; particularly vital for those whose views might most readily be discounted (O'Brien 1987). Independence from service provision is, therefore, crucial (Barnes \& Brandon 2002). IMHA services are a form of statutory advocacy and can be instructed or non-instructed, where the advocacy partner is unable to instruct the advocate directly. It is framed as a necessary safeguard within the context of legitimised compulsion to protect rights and ensure service user voices are heard in decisions about care and treatment (Department of Health 2008).

The MH Act reforms have been described as an unhappy combination of legalism and safeguards, and stand accused of maintaining state-sanctioned coercion (Pilgrim 2012). Such critique has been bolstered by increasing rates of detention, a perceived decline in therapeutic standards in inpatient care (CQC 2011a, 2012, Pilgrim 2012), and consistent evidence that some groups, especially black minority ethnic (BME) communities, have higher overall detention rates (CQC 2010, 2011a,b, 2012, 2014), negative experiences of care and poorer outcomes (Morgan 2012). The introduction of IMHA services was viewed by some as a response to prolonged opposition in the course of the $\mathrm{MH}$ Act reform process, and the introduction of CTOs, while welcomed by others as a step in the right direction for service user rights (Mental Health Alliance 2008).

Potential shortfalls in advocacy services to meet anticipated needs for access to advocacy under the Mental Health (Care \& Treatment) (Scotland) Act, 2003 were expressed prior to major reform of mental health law (Grant 2004). In England, a year after the implementation of the statutory right to IMHA services, one in five wards did not have access (CQC 2010, 2011a), and only a minority had support from an IMHA in the tribunal process (Administrative Justice and Tribunals Council and CQC 2011). Concerns about awareness of entitlement to advocacy by service users (Newbigging et al. 2007, 2011) and by professionals, who have a duty to explain access to IMHA services to qualifying patients, have been 
implicated in limiting uptake (CQC 2010, 2011a,b, 2012, 2014).

Furthermore, issues have been identified in relation to equity of access with gaps in provision of generic mental health advocacy for minority groups, and those with other specific needs (Foley \& Platzer 2007). For people from BME communities, this reflects shortcomings in provision: funding for BME advocacy, understanding among mainstream mental health advocacy services of cultural issues, and partnership working between advocacy services and BME organisations (Rai-Atkins et al. 2002, Newbigging et al. 2007, 2011). The rapid pace of introduction of IMHA services has fostered inequities in access, with BME organisations disadvantaged by commissioner pragmatism in rolling over existing contracts (Hakim \& Pollard 2011).

Research and service user accounts indicate that once people access mental health advocacy, it is appreciated and leads to a greater sense of wellbeing, self-efficacy and empowerment (Mind 2006, Palmer et al. 2012). In a case-control study of an experimental model of advocacy compared with statutory advocacy in Australia (Rosenman et al. 2000), the person-centred model reduced the risk of future detention, and improved attendance for aftercare and patient experience of detention. A small-scale study of IMHA services in Bexley, London (Palmer et al. 2012) highlighted the importance of the style of provision in promoting trust and positive engagement with service users, resonating with findings from earlier studies (Foley \& Platzer 2007, Newbigging et al. 2011). The extant research, therefore, raises questions about access to equitable, appropriate, effective and good-quality IMHA services.

\section{Aim}

The overarching aim of the research was to: (i) review the extent to which IMHA services in England are providing accessible, effective and appropriate advocacy support to the diversity of qualifying patients and (ii) identify the factors that determine the quality of IMHA services, from service user, commissioner and provider perspectives.

\section{Research design and methods}

A multi-method approach was deployed, with two stages of data collection undertaken between December 2010 and November 2011. The aim of Stage 1 was to define what constitutes high-quality IMHA services; a prerequisite to evaluating effectiveness at a local level (Donabedian 2002). This involved a focused literature review, observations of practice in 4 advocacy services and 11 focus groups. The focus groups were distributed across England and were targeted at different stakeholders, with the exception of two focus groups for a mix of participants. A total of 75 participants were recruited via local networks (see Table 1).

A focus group for commissioners was planned, but they proved difficult to recruit, reflecting the uncertainty facing Primary Care Trusts in late 2010. The lines of enquiry for the focus groups are summarised in Figure 1.

Draft indicators for the quality of IMHA services were developed from synthesising data from Stage 1, to identify the factors that influenced the quality of IMHA services. These were refined through discussion with an expert stakeholder group and used to guide data collection during Stage 2, as summarised in Figure 2.

A case study design was adopted in Stage 2, enabling the different dimensions of quality, identified from Stage 1, to be explored across a variety of practice contexts (Yin 2008). Information on the use of the $\mathrm{MH}$ Act was reviewed to identify potential sites (i.e. MH Trusts), and additional consideration given to the organisational arrangements for IMHA provision [generic/mental health/alongside Independent Mental Capacity Advocacy (IMCA)/BME/size]

Table 1 Focus group participants

\begin{tabular}{lc}
\hline Participants & Participants $(N=75)$ \\
\hline Service users & $\begin{array}{c}\text { Eighteen participants: one focus group for BME mental health service users ( } n=5, \text { London); } \\
\text { two focus groups for IMHA partners }(n=3, \text { East of England and } n=5, \text { North West), two } \\
\text { mixed stakeholder groups }(n=1, \text { Yorkshire and } n=1, \text { South West) plus three telephone interviews }\end{array}$ \\
$\begin{array}{l}\text { Family carers } \\
\text { IMHA providers }\end{array}$ & $\begin{array}{l}\text { Forty-one participants in four focus groups for IMHAs, including one for BME advocacy } \\
\text { providers (North West and London) and in two mixed stakeholder groups (Yorkshire and South West) }\end{array}$ \\
Mental health staff & Six participants in a focus group for mental health staff (North West) \\
Commissioner & One participant in a mixed stakeholder focus group (Yorkshire)
\end{tabular}

IMHA, Independent Mental Health Advocate. 


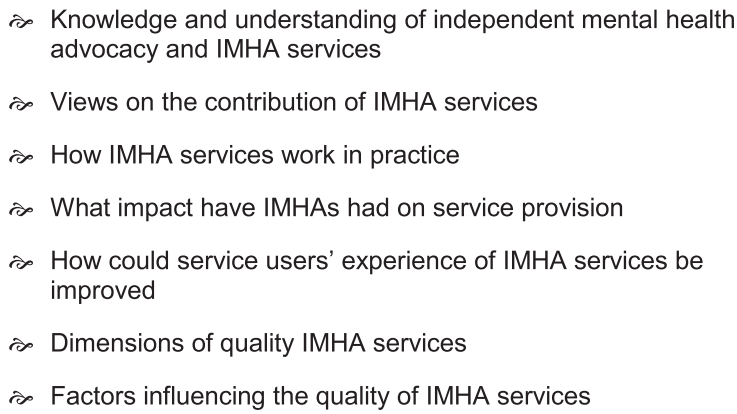

Figure 1 Focus group lines of enquiry. IMHA, Independent Mental Health Advocate.

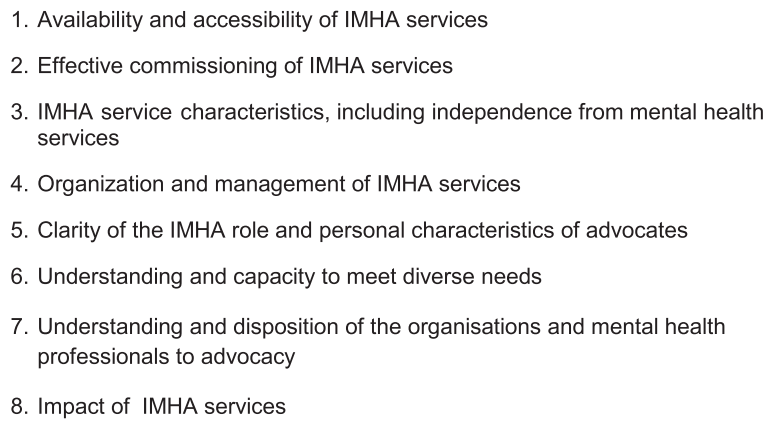

Figure 2 Draft indicators identified from Stage 1. IMHA, Independent Mental Health Advocate.

and mental health provision. Efforts were made to also ensure geographical spread across England. The final eight sites included:

- Two NHS mental health Trusts in inner city locations.

- Two NHS mental health Trusts in urban locations.

- Two NHS mental health Trusts in a mix of suburban and rural, including coastal areas.

- One independent provider of medium and low secure services for people placed out of area.

- One NHS provider of high, medium and low secure services.

In each of the case study sites, a particular focus was adopted: either in the selection of the initial case study site (for example identifying an independent sector provider to look at IMHA provision for people placed out of area) or by targeted recruitment of participants (for example, people from BME communities). This enabled IMHA provision to be considered:

- Across the age spectrum.

- For diverse communities.

- For people on CTOs.
- In settings with different levels of security (from low to high).

- For people placed out of area

In each site, the following data collection methods were used to explore both the operation and experience of IMHA services:

- A survey questionnaire: all advocacy providers in the area of the case study sites $(n=22)$ were invited to complete the survey questionnaire online. The questionnaire sought to elicit service principles, current organisational arrangements, resources available and caseload. Responses to the questionnaire from a total of 18 of a potential 22 services were received, giving a response rate of $82 \%$.

- Analysis of key documentation including service specifications, engagement protocols and reports from IMHA providers.

- Semi-structured qualitative interviews with 20-25 participants purposively selected from different stakeholder groups, and a range of recruitment methods used to ensure a broad range of perspectives were sampled. Figure 3 provides a summary of the key questions, which were tailored to each participant group (e.g. IMHA partner, professional, IMHA, etc.).

- Case note analysis of IMHA records and mental health records for a small sub-sample of consenting qualifying patients $(n=20)$ to investigate the impact of IMHA activities on care and treatment under the MH Act.

\section{Service user involvement}

A key feature of this research was the partnership with mental health service users making up three quarters of the research team (10 of 13 researchers), with shared experience of inpatient detention, including secure services. A range of skills and experiential knowledge was drawn upon, and we endeavoured to

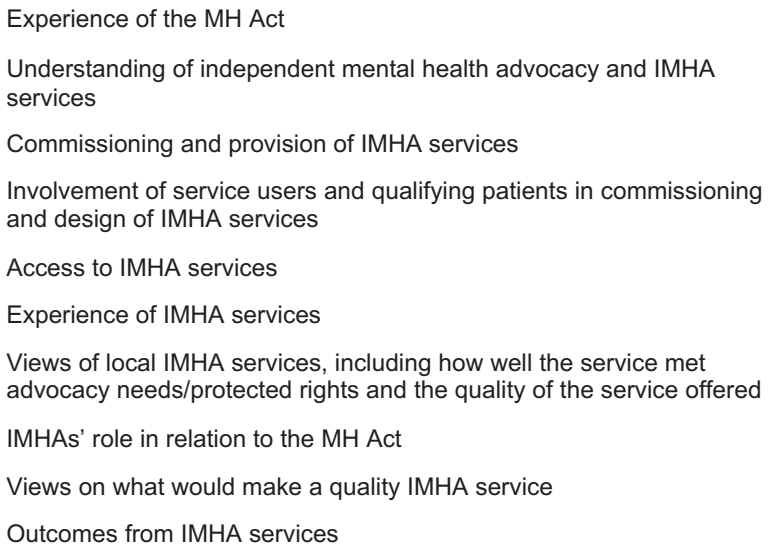

Figure 3 Topic guide for semi-structured interviews. MH, Mental Health; IMHA, Independent Mental Health Advocate. 
be consciously reflexive about how this shaped our approach to the research (Newbigging et al. 2012).

\section{Data analysis}

Data from focus groups and interviews were analysed using standard qualitative methods, beginning with identification of key themes and patterns (Coffey \& Atkinson 1996, Silverman 2011) informed by the research objectives and team members' interpretations. NVivo 9 software supported a process of systematic data analysis, enabling comparisons between different data sources and case study sites, and further investigation of emergent themes. Summary statistics were produced from the survey questionnaire data and, wherever possible, comparison made with national data sets [e.g. Mental Health Minimum Data Set (MHMDS), Health and Social Care Information Centre 2011] to estimate how many qualifying patients were accessing IMHA services across the sites for 2010-2011. The findings from all sources of data were synthesised, and relationships between advocacy needs, organisational context, uptake of advocacy services, front-line practice, and experiences of IMHA services were examined.

\section{Ethical approval}

Ethical approval for the study was granted by Cambridgeshire 3 Research Ethics Committee and the University of Central Lancashire Ethics Committee, with local research governance negotiated at each of the eight sites. The main ethical issues related to consent, confidentiality and potential distress to participants. In relation to consent, all service user participants were assumed to be competent to consent unless there was specific evidence this was not the case (HMSO 2005). In two instances of a young person being under the age of 16, parental consent was sought. In the event of participants becoming distressed, they were given the option of terminating the interview and all participants were offered information about sources of support, including advocacy services, at the end of the interview. Data were anonymised and kept secure consistent with the University's data protection policy.

\section{Findings}

\section{Study participants}

There were a total of 289 participants: 75 participating in focus groups at Stage 1 (see Table 1) and 214 in interviews in Stage 2 (see Table 2).
One hundred and five qualifying patients were recruited, but seven people subsequently withdrew and a further eight were judged to lack capacity to consent. Ninety qualifying patients were, therefore, interviewed; 61 with experience of using IMHA services. The majority $(n=82)$ completed a questionnaire to provide demographic information (see Table 3). This indicates that most participants $(n=75)$ had been in contact with mental health services for more than 1 year, and had been sectioned more than once.

The themes identified from the data analysis are reported here with reference to the study objectives concerning availability and access, appropriateness and effectiveness of IMHA services. This section starts by locating these in data on need for IMHA services and concludes with the findings in relation to the mental health services context in which IMHA services operate.

\section{Need for IMHA services}

The dominant theme from service user accounts was of the process of admission as bewildering, and detention under the $\mathrm{MH}$ Act as frightening and disempowering. Instances where people had been forcibly admitted or received medication against their will were relayed:

I tried to leave home and then they [parents] rang the doctors. The doctors rang the police, and they came to collect me from the train station, I was brought in here in a police van. I wasn't a criminal, I wasn't dangerous, I wasn't hitting anybody or being a threat. (IMHA partner, Acute Ward)

Qualifying patients identified 'being treated with humanity' as having the potential to change the nature of their experience, but personal views on recovery were jeopardised by a focus limited to medical treatment. One man, for example, considered dismissal of his views had led him being in hospital much longer than needed. Accordingly, once qualifying patients who had not used IMHA services gained an understanding of the role, they considered it would have been helpful and requested information about how to access local services:

They need to find some sense, these people are going through turmoil, so what they actually need is some kind of link, some way to hold onto something. (Non-IMHA user, Acute ward)

Table 4 provides a comparison of the reasons for referral to IMHAs by participant type, highlighting the importance for qualifying patients of having a voice and coming off their section: 


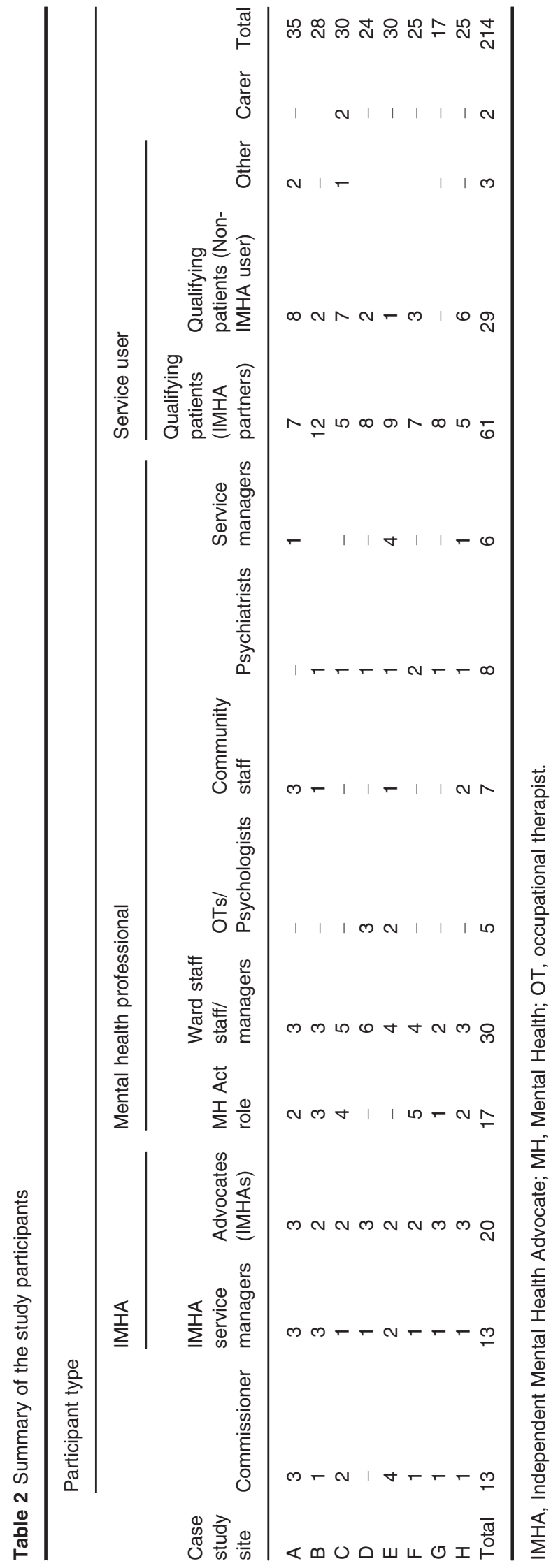

No matter how coherent, how rational, how considered my arguments and my explanations for events are, that assumed level of incompetence undermines it because you're given sub status. (IMHA partner, Acute ward)

The case note analysis indicated that mental health professionals rarely recorded referral to or contact with IMHA services, but revealed instances where IMHA involvement could have strengthened shared decision-making and/or influenced outcomes, for example in relation to hospital leave or medication.

\section{Availability and access}

Comparing data from the survey questionnaire of IMHA services with the MHMDS, we found that overall less than half of qualifying patients had accessed IMHA services. This ranged from 19\% (264 people of 1370 detained patients) in an inner city site to $92 \%$ (210 of 228 detained patients) in a site providing secure services. For people on CTOs, the uptake was generally lower, with a range from $5 \%$ to $55 \%$ across the sites. These findings need to be interpreted cautiously because of issues with data quality reflecting the accuracy of information kept by IMHA services. It does, however, suggest some interesting trends. First, access was easiest for those in secure services, with more static populations, where relationships with IMHA providers were more established, and protocols for automatic referral on admission were routine. Access and uptake were lower in the two inner city study sites reflecting high occupancy and turnover rates, and complexity of need. Slightly more men than women used IMHAs across the case study sites and uptake from BME service users was generally low. The relatively low uptake by children and young people reflected differences in commissioning and whether a specific service was available. Similarly, uptake for older people, particularly those requiring noninstructed advocacy, was higher in sites where relevant expertise had been developed, with only one advocacy provider in one site having a specific service for older people with dementia. Across the sites, IMHAs and commissioners interviewed also suggested that access was problematic for people subject to CTOs and those placed out of area.

The average caseload size of individual IMHAs was 25 cases, but this varied markedly from 8 to 55 cases across the sites, and did not appear to reflect the number of IMHAs in a service. From interviews with commissioners and documentary analysis, we found little evidence that resourcing decisions were based upon systematic needs assessment. For example, the implications of national statistics showing 
Table 3 Characteristics of the sample of qualifying patients

\begin{tabular}{ll}
\hline Dimension & Sample characteristics, $n(\%)$ \\
\hline Age & Under $21: 11(13 \%)$ \\
& $22-65: 65(80 \%)$ \\
& Over $65: 5(6 \%)$ \\
Ethnicity & White British: $57(70 \%)$ \\
& $25(30 \%)$ from a BME community, including black Caribbean $8(10 \%)$, black African $4(5 \%)$, \\
& Mixed heritage $3(4 \%)$, South Asian $4(5 \%)$, Chinese $1(1 \%)$, white Irish $2(3 \%)$ with the \\
English as the first spoken language & remainder being white European, white Australian or other mixed heritage $3(4 \%)$ \\
Sexual orientation & Seventy-two participants $(88 \%)$ \\
Disability & Fifty-three participants $(65 \%)$ identified having a disability with $29(35 \%)$ defining this \\
& as a mental health condition and 10 (12\%) a learning disability or autistic spectrum disorder \\
Contact with mental health services & Less than 1 year: $7(9 \%)$ \\
& $1-5$ years: $27(33 \%)$ \\
Number of times sectioned & More than 5 years: $48(58 \%)$ \\
& $1: 21(26 \%)$ \\
& $2: 14(17 \%)$ \\
& $>3: 41(50 \%)$ \\
& No response: $(6) 7 \%$ \\
\hline
\end{tabular}

Table 4 Main reason for referral to IMHA services by referrer type (rank ordered)

Referral source

\begin{tabular}{|c|c|c|}
\hline Self & Mental health professionals & IMHAs \\
\hline $\begin{array}{l}\text { (1) To have a voice } \\
\text { (1) Coming off section }\end{array}$ & (1) Rights under the MH Act & (1) Ward rounds. CPA/Tribunals \\
\hline $\begin{array}{l}\text { (2) Negative experiences of care } \\
\text { and treatment }\end{array}$ & $\begin{array}{l}\text { (2) No family or in conflict with the family } \\
\text { (2) Complaints/disagreement with care plan }\end{array}$ & (2) Leave or being discharged \\
\hline $\begin{array}{l}\text { (3) Tribunal, managers' meetings, } \\
\text { CPA and ward rounds } \\
\text { (4) Information and support }\end{array}$ & $\begin{array}{l}\text { (3) Person not well enough to self-advocate/lacking capacity } \\
\text { (3) Determine appropriate care to facilitate recovery } \\
\text { (4) Move to a different service or accommodation }\end{array}$ & $\begin{array}{l}\text { (3) Rights under the MH Act } \\
\text { (4) Medication }\end{array}$ \\
\hline (5) Medication & (5) Lack of trust in staff & (5) Safeguarding/vulnerability \\
\hline
\end{tabular}

IMHA, Independent Mental Health Advocate; MH, Mental Health; CPA, Care Programme Approach.

disproportionate rates of occupancy relating to specific BME communities (e.g. CQC 2011b) had not been considered.

Access was also influenced by the limited awareness and confusion about IMHA services among qualifying patients and professionals. Over two-thirds of qualifying patients interviewed, who had not used IMHA, did not know what it was for, confused its purpose with complaints advocacy, or did not know what the service could do for them. This contrasted with explanations for low uptake offered by professionals, generally attributing this to service user choice. The quality of promotional materials was variable, some of which were out of date, and qualifying patients wanted more information, including pictures of local advocates, so they could be easily recognised and examples of how an IMHA could help them. Mental health status, circumstances surrounding hospital detention and the sheer information overload experienced when being detained under the MH Act could also serve as barriers to access.

Attitudes to advocacy and the extent to which mental health professionals fully understood the IMHA role varied across the sites and in different settings within the same study sites. In general, professionals working in secure services were more likely to understand their obligations to promote access to IMHA services. In one site, only one in five of the staff interviewed understood their obligations, and confusion with the IMCA role was common with some believing IMHA services were only for detained patients lacking capacity. Thus, access to IMHA 
services could be limited by professionals promoting the service for people lacking capacity or for those who did not have family carers (as noted in Table 4). Misunderstandings reflected the scant training the majority of staff had received, with the exception of Approved Mental Health Practitioners. Those that did have a good grasp of the role appreciated the value of IMHAs providing 'checks and balances' in the system.

Qualifying patients, IMHAs and a minority of mental health professionals identified IMHAs making regular visits to wards as a way of overcoming these potential barriers to access and uptake.

Familiarity brings down the barriers for people to want to trust him because it's a big step. So the IMHA coming on the unit regularly has brought down barriers and people now talk to him about various issues and problems so that's very good. (IMHA partner)

IMHAs identified the lack of resources and demands on their service as limiting the opportunities for this. Family carers in the focus group, although largely unaware of IMHA services, highlighted the role they could potentially play in promoting awareness and access.

\section{Appropriateness of support}

On the whole, once people accessed IMHA services, they were satisfied and appreciated the support they received, deeming it to be relevant and suitable to their needs. There was, however, a general lack of culturally appropriate services, going beyond addressing language barriers to having a deeper appreciation of salient issues, particularly experiences of racism and associated lack of trust in mental health services. A notable exception was a service in an inner city site, developed as part of a range of services designed to support local BME communities. Gaps in provision for qualifying patients with learning difficulties, and people who are deaf or hearing impaired were identified, and in some instances, advocacy was being provided by community organisations not funded, or trained, to do so. Although strategies to engage particular groups or partnership working with community organisations were identified as facilitating both better access and more appropriate service delivery, they were rare in practice.

IMHA providers were undertaking equalities monitoring, although this was largely restricted to gender, age and ethnicity, with some unease about capturing data on sexual orientation. IMHA providers typically stated that their person-centred approach enabled them to respond flexibly to a diverse range of needs. However, IMHA partners were rarely being involved in the design or evaluation of IMHA services. Approximately, three quarters of the IMHA workforce were white women, making it difficult to offer a choice of advocate. That said, most qualifying patients valued relational qualities and effectiveness of support above specific demographical characteristics of the advocate.

Availability of different types of advocacy, instructed or non-instructed, is also important. Few services had IMHAs who were also trained as IMCAs. Further, many IMHAs were uncomfortable acting in the absence of instruction from an advocacy partner, doing all they could to establish the individual's wishes and preferences. Consequently, provision of non-instructed IMHA was uncommon, pointing to a gap in provision, particularly for older people with dementia.

The important interface between IMHA and generic advocacy raised dilemmas, with people often needing support in expressing views other than pertaining directly to the MH Act, but relevant to their recovery journey or broader health and social needs. Concern was raised by IMHAs in focus groups, that the statutory IMHA role had created unhelpful boundaries to advocacy practice that in some instances impeded a more holistic approach, with informal or voluntary patients losing out. This was not evident, however, in practice in the case study sites: rather IMHAs were working flexibly to be responsive:

It's a continuum, I see this person in the community, they've got an issue, I help them resolve it. The next time I see them they're an informal patient. They still see me because they're on our database as having been referred to me before so they automatically see me ... Six months, a year goes by they're actually admitted on section. I'm the IMHA who sees them because they've already been referred to me. (IMHA)

Whether or not the role of IMHAs in Tribunals and managers' meetings was always appropriate was raised both by IMHAs and mental health professionals. For some, there was concern that IMHAs were becoming quasi-legalistic and indeed sometimes substituting for legal representation at Tribunals because of changes in legal aid entitlement. By default, this adversely influenced Tribunal chairs and panels' expectations and understanding of the IMHA role.

\section{Effectiveness}

IMHA services and commissioners routinely monitor service user satisfaction with IMHA services. However, routine reporting of outcomes from IMHA interventions was less common. From the service user 
perspective, IMHAs played an invaluable role in ensuring their voice was heard in the system. Across the case study sites, they were variously described by advocacy partners in an active role of 'godmotherly person', a 'lever' or a 'hammer' because they made something happen. Others described the negotiating, smoothing role as a 'diplomat' or 'bridge' between service users and mental health professionals, even being the 'WD40' in the system. For some, IMHA services had been life changing, while these were of limited benefit for others. From these accounts, we identified a distinction between the impact of the advocacy process and achieving tangible outcomes, summarised in Figure 4.

Although a concrete outcome (such as changing the level of restriction, or lifting the section) might not be what was achieved, there was a high level of satisfaction with involving IMHAs:

It's not changed anything that's happening here at all ... [But] it's made me feel better within myself because people are treating me as a human being and not a bit of dirt under their feet ... It gives you confidence within yourself. (IMHA partner, Rehab unit)

IMHA partners identified improved communication with the care team as a key outcome, and the sheer presence of an advocate could alter staff behaviour positively; opening up clinical environments to wider scrutiny:

Once a week two advocates come to the ward. It opens it up, it makes things transparent... it changes the dynamic ... opening this place ... the more light comes on it, the more open and transparent it becomes ... [Staff] are being a little bit more careful, a little bit more wary ... (IMHA partner, Acute ward)

A minority of IMHA partners felt an IMHA had been helpful in enabling them to come to terms with their situation, and this would benefit them in the longer term, while a smaller number thought that instead of empowering them, it had served to main- tain the status quo. Alternately, a lack of positive impact was attributed by IMHA partners to unresponsive care services, while IMHAs also highlighted the complexity of some people's circumstances as mitigating factors.

\section{Mental health services context}

As noted earlier, there was considerable variation among professionals in understanding their duty to promote access to IMHA, and their role in shaping the context in which IMHAs operate. Some professionals actively promoted IMHA and provided a supportive context, while others viewed IMHA input as counterproductive to clinical interventions and patients' 'best interests':

Sometimes the advocates have been pressing a little harder than they should be for things like obtaining leave when they have been explained you know it is not possible, or it is not in the interest of the patient to give leave, but they still insist. (Psychiatrist)

Such misunderstanding could lead to fractious relationships between professionals and IMHAs, for example, not negotiating times of key meetings so the IMHA could attend. Conversely, effective working relationships reflected a mutual understanding of roles and expectations and the constraints each work under. In these circumstances, professionals drew a clear distinction between independent advocacy and 'best interests', and perceived challenges to professional opinion were met with equanimity.

\section{Discussion}

Our findings are consistent with the CQC's critique of access issues (CQC 2010, 2011a, Kinton 2014): those requiring IMHA services the most may make least use of them. Similarly, Hakim and Pollard (2011) described gaps in IMHA services for people

\begin{tabular}{|l|l|}
\hline \multicolumn{1}{|c|}{ Process } & \multicolumn{1}{c|}{ Outcomes } \\
\hline - Supportive and empowering approach & - Increased understanding and knowledge \\
'Someone alongside on your side' & of rights and treatment \\
- Empowering service users to have a & - Reduction in length of detention \\
voice & - Increased sense of well-being \\
- Increasing confidence to advocate & - Helped towards service users' recovery \\
- Providing information to increase & by increased sense of personal agency \\
understanding of rights and treatment & - Empowered to exercise rights \\
- Helping navigate the service territory & - Increased participation in decisions \\
- Performing an important safeguarding & affecting personal care and treatment \\
function by providing independent & - Acceptance of the status quo \\
scrutiny of the relationship with & - Successful resolution of complaints \\
professionals & \\
\hline
\end{tabular}

Figure 4 Outcomes identified by Independent Mental Health Advocate partners. 
from BME communities. Our findings go further in identifying gaps for other groups: namely older people with dementia, people with learning disabilities, people with sensory disabilities, people on CTOs and young people. We suggest three reasons for this: (i) barriers in terms of lack of awareness of, or trust in, advocacy services, (ii) mental health professionals acting as gatekeepers and (iii) a model of advocacy practice that reflects origins within the largely white service user movement, grounded in instructed advocacy. This does not sit comfortably with a conception of advocacy targeting those most at risk of exclusion (O'Brien 1987) or the spirit of the MH Act 2007, promoting access to IMHA services as a universal right.

There are two potential remedies. First, automatic referral of qualifying patients to IMHA services on compulsion, offering the opportunity to opt-out rather than opt-in (House of Commons Select Committee 2013). Second, to strengthen the overall advocacy presence, particularly on inpatient wards, by extending IMHA provision to all inpatients (see National Institute for Health \& Clinical Excellence 2011, Welsh Government 2011). This should be linked to provision of timely and understandable information, facilitating access to the most appropriate form of advocacy.

The inter-relationship between commissioning, IMHA services and mental health services crucially influences quality. An over-reliance on standards and codes of practice for IMHA providers alone will be of limited impact. There is an increasing trend towards commissioning large national organisations to provide IMHA services, but the benefits of an established infrastructure and profile need to be weighed against the social capital of community-based organisations, arguably more effective in increasing uptake for particular groups (Newbigging et al., 2008).

Appreciation and understanding of advocacy in general, and IMHA in particular, shape professionals' response (Lacey \& Thomas 2001, Carver \& Morrison 2005). Professionals were by no means universally antagonistic to IMHA services, but this was more likely to be the case if they saw advocacy in terms of 'best interests' rather than supporting voice and empowerment. Thus, it becomes possible to distinguish the readiness of various care teams to engage with advocacy (McKeown et al. 2014). Service users and mental health professionals recognise that the advocate's presence can alter the dynamic towards shared decision-making. IMHA services are undoubtedly valued by advocacy partners even if they do not realise tangible benefits, with a distinction between process and outcomes of advocacy
(Townsley et al. 2009). The general lack of evaluative research suggests a need for further work on outcomes.

Finally, our findings (see also Rosenman et al. 2000) raise questions about the potential limitations of a narrow focus on the statutory role. An emphasis on negative rights or 'procedural rights', i.e. the right to refuse treatment and freedom from detention (Gostin 2000, Carney et al. 2008), can neglect more substantive rights such as the right to health (Battams \& Henderson 2010). This potentially limits the effectiveness of IMHA provision and generates role conflict for IMHAs. We should not, however, lose sight of the extent to which advocacy can enhance personal agency, supporting hope and recovery amidst debilitating life circumstances (Shepherd et al. 2010, Maddock \& Hallam 2010). With growing numbers of people subject to compulsion, it would be foolhardy to dismiss IMHA as a mere palliative. Rather, a cleareyed view of its limitations is needed, as is strengthening its role in empowering individuals to be active agents in their own care. The standpoint adopted by services will depend on the context or willingness of staff to embrace the challenge of advocacy.

\section{Limitations}

It is not possible to confirm how representative the eight case study sites were, although the comprehensive nature of the study increases confidence that the major themes were identified. Data on access should be interpreted cautiously as the mental health providers or IMHA services, with one exception, did not share information about qualifying patients. We encountered practical challenges in relation to participant recruitment: consequently, there are some gaps in the participant profile, for example older people and carers. We also did not observe as many instances of non-instructed advocacy as we had hoped. Furthermore, the research was undertaken relatively early and some of the issues highlighted may reflect implementation problems. These limitations suggest avenues for further research.

\section{Conclusion}

If IMHA is to offer a sense of agency for those whose freedoms are constrained, the conception of IMHA needs to move beyond a narrow focus on safeguarding rights to a broader remit promoting self-determination and participation in decisions about care and treatment, opening up horizons beyond legal detention. Operationalising this will depend on a culture shift to a rights focus, evidenced by informed 
commissioning - of accessible and appropriate IMHA services built on an understanding of diversity - and a mental health service context, supportive of advocacy principles. In the absence of attention to these different dimensions and a systematic approach to quality, there is a distinct risk that the IMHA role will focus on procedural rights, potentially serving to reinforce the status quo, and opportunities to maximise self-determination and promote recovery will be missed.

\section{Acknowledgements}

This study was funded by the Department of Health Policy Research Programme. The views expressed are not necessarily those of the Department. This study was undertaken in partnership with Laura Able, Kaaren Cruse, Paul Grey, Zemikael Habte-Mariam, Stephanie de la Haye, Doreen Joseph, Michelle Kiansumba and June Sadd.

\section{Conflict of interests}

None.

\section{References}

Administrative Justice and Tribunals Council and the Care Quality Commission (2011) Patients' Experiences of the First-Tier Tribunal (Mental Health): Report of a Joint Pilot Project of the Administrative Justice and Tribunals Council and the Care Quality Commission. Care Quality Commission, Newcastle.

Barnes M. (2007) A final brick in the wall? A history of the Nottingham Advocacy Group. Available at: http://eprints. brighton.ac.uk (accessed on 22/10/2013).

Barnes D. \& Brandon T. (2002) Independent Specialist Advocacy in England and Wales: Recommendations for Good Practice. University of Durham, Durham.

Battams S. \& Henderson J. (2010) The physical health of people with mental illness and 'the right to health'. Advances in Mental Health 9, 116-129.

Carney T., Tait D. \& Beaupert F. (2008) Pushing the boundaries: realising rights through mental health tribunal processes. Sydney Law Review 30, 329.

Carver N. \& Morrison J. (2005) Advocacy in practice: the experiences of independent advocates on UK mental health wards. Journal of Psychiatric and Mental Health Nursing 12, 75-84.

Coffey A.J. \& Atkinson P.A. (1996) Making Sense of Qualitative Data: Complementary Research Strategies. Sage, London.

CQC (2010) Mental health act annual report 2009/10. Available at: http://www.cqc.org.uk/public/reports-surveys-and-reviews/reports/mental-health-act-annual-report2010/11 (accessed on 23/10/2013).

CQC (2011a) Mental health act annual report 2010/11. Available at: http://www.cqc.org.uk/public/reports-surveysand-reviews / reports / mental-health-act-annual-report-2010/ 11 (accessed on 22/10/2013).
CQC (2011b) Count Me In 2010: Results of the 2010 National Census of Inpatients and Patients on Supervised Community Treatment in Mental Health and Learning Disability Services in England and Wales. Care Quality Commission, London. Available at: http://www.cqc.org.uk/content/count-me2010-census (accessed on 23/10/2013).

CQC (2012) Mental Health Act Annual Report 2011/12. Care Quality Commission, Newcastle.

CQC (2014) Monitoring the Mental Health Act in 2012/13. Care Quality Commission, Newcastle.

Department of Health (2008) Code of Practice: Mental Health Act 1983. TSO, Norwich.

Department of Health (2012) Transforming care: a national response to Winterbourne View hospital. Available at: https://www.gov.uk/government/publications/winterbourne-view-hospital-department-of-health-review-andresponse (accessed on 23/10/2013).

Donabedian A. (2002) An Introduction to Quality Assurance in Health Care. Oxford University Press, Oxford.

Foley R. \& Platzer H. (2007) Place and provision: mapping mental health advocacy services in London. Social Science and Medicine 64 (3), 617-632.

Gostin L.O. (2000) Human rights of persons with mental disabilities: the European Convention of Human Rights. International Journal of Law and Psychiatry 23 (2), 125159.

Grant S.E. (2004) National Mental Health Services Assessment: Towards Implementation of the Mental Health (Care and Treatment) (Scotland) Act 2003: Final Report. Scottish Executive, Edinburgh.

Hakim R. \& Pollard T. (2011) Independent mental health advocacy: briefing paper 3. Mental Health Alliance. Available at: http://www.mentalhealthalliance.org.uk/resources/Independent_Mental_Health_Advocacy_report.pdf (accessed on $23 / 10 / 2013$ ).

Health and Social Care Information Centre (2011) Fifth report from Mental Health Minimum Dataset (MHMDS) annual returns. Available at: http://www.hscic.gov.uk/ article/2021/ (accessed on 23/10/2013).

Henderson R. \& Pochin M. (2001) A Right Result? Advocacy, Justice and Empowerment. The Policy Press, Bristol.

HMSO (1983) Mental Health Act 1983. Her Majesty's Stationery Office, London.

HMSO (2005) Mental Capacity Act 2005. Her Majesty's Stationery Office, London.

House of Commons Select Committee (2013) First Report Post-legislative scrutiny of the Mental Health Act 2007.

Kinton (2014) An Audit of Data from MHA Commissioner Visits July-December 2013. Presentation at Commissioning Independent Mental Health Advocacy services: What does good look like? NCVO, London, February 19.

Lacey Y. \& Thomas P. (2001) A survey of psychiatrists' and nurses' views of mental health advocacy. Psychiatric Bulletin 25, 477-480.

Maddock S. \& Hallam S. (2010) Recovery Begins with Hope. National School of Government, London.

McKeown M., Ridley J., Newbigging K., Poursanido D., Machin K. \& Cruse K. (2014) A conflict of roles, a conflict of ideas? The unsettled relations between care team staff and independent mental health advocates. International Journal of Mental Health Nursing 23 (5), 398-408.

Mental Health Alliance (2008) New mental health powers must be used wisely, says Alliance. Available at: http://www. 
mentalhealthalliance.org.uk/news/prpowersusedwisely. $\mathrm{html}$ (accessed on 23/10/2013).

Mind (2006) With Us in Mind: Service User Recommendations for Advocacy Standards in England. Mind, London.

Morgan C. (2012) 10 Years On: Ethnicity and the Long-Term Course and Outcome Psychosis. Presentation to the MHRN 2012 National Scientific Meeting, Birmingham, 25 April 2012.

NIHCE (2011) Service user experience in adult mental health: improving the experience of care for people using adult NHS mental health services: Clinical Guideline 136. National Institute for Health and Clinical Excellence. Available at: http:/ / guidance.nice.org.uk/CG136/NICEGuidance/pdf/ English (accessed on 23/3/2014).

National Institute for Mental Health in England (2008) Independent mental health advocacy: guidance for commissioners. Available at: http://nmhdu.org.uk/.../files/independentmental-health-advocacy-guidance.pdf (accessed on 23/ 10/2013).

National Mental Health Development Unit (2009) Independent mental health advocacy: effective practice guide. Available at: http://www.nmhdu.org.uk/silo/files/independent-mental-health-advocacy-effective-practice-guide. pdf (accessed on 23/10/2013).

Newbigging K., McKeown M., Hunkins-Hutchinson E. \& French B. (2007) Mtetezi: Mental Health Advocacy with African and Caribbean Men. Social Care Institute for Excellence, London.

Newbigging K., McKeown M., Habte-Mariam Z., Mullings D., Charles J.J. \& Holt K. (2008) Commissioning and Providing Mental Health Advocacy for African and Caribbean Men: A Resource Guide. London, Social Care Institute for Excellence. Available at: http://www.scie.org.uk/publications/guides/guide21/index.asp (accessed 1/9/14)

Newbigging K., Ridley J., McKeown M., Machin K. \& Poursanidou D. (2012) The Right to Be Heard: Review of the Quality of IMHA Services. Report for the Department of Health. UCLan, Preston.

Newbigging K., McKeown M. \& French B. (2013) Mental health advocacy and African and Caribbean men: good practice principles and organizational models for delivery. Health Expectations 16(1), 80-104.

NHS Information Centre (2011) Mental health bulletin: fifth report from mental health minimum data set (MHMDS) annual returns 2011. Available at: http://www.ic.nhs.uk/ pubs/mhbmhmds11 (accessed on 14/4/2012).
O'Brien J. (1987) Learning from Citizen Advocacy Programs. Georgia Advocacy Office, Atlanta, GA.

Palmer D., Nixon J., Reynolds S., Panayiotou A., Palmer A. \& Meyerowitz R. (2012) Getting to know you: reflections on a specialist independent mental health advocacy service for Bexley and Bromley residents in forensic settings. Mental Health Review Journal 17 (1), 5-13.

Pilgrim D. (2012) Lessons from the Mental Health Act. Commission for England and Wales: the limitations of legalism-plus-safeguards. Journal of Social Policy 41 (01), 61-81.

Rai-Atkins A., Jama A.A., Wright N., Scott V., Perring C., Craig G. \& Katbamma S. (2002) Best Practice in Mental Health: Advocacy for African, Caribbean and South Asian Communities. Policy Press, Bristol.

Randall D. \& McKeown M. (2014) Editorial: Failure to care: nursing in a state of liquid modernity? Journal of Clinical Nursing 23, 766-767.

Rosenman S., Korten A. \& Newman L. (2000) Efficacy of continuing advocacy in involuntary treatment. Psychiatric Services 51 (8), 1029-1033.

Shepherd G., Boardman J. \& Burns M. (2010) Implementing Recovery. Sainsbury Centre for Mental Health, London.

Silvera M. \& Kapasi R. (2002) A Standards Framework for Delivering Effective Health and Social Care Advocacy for Black and Minority Ethnic Londoners; A Consultancy Partnership Project by SILKCAP and OPM Funded by the King's Fund. King's Fund, London.

Silverman D. (2011) Interpreting Qualitative Data. Sage, London.

Townsley R., Marriott A. \& Ward L. (2009) Access to Independent Advocacy: An Evidence Review. Office for Disability Issues, London.

Wallcraft J. \& Nettle M. (2009) History, context \& language. In: J. Wallcraft, B. Schrank \& M. Amering (Eds) Handbook of Service User Involvement in Mental Health Research, pp. 1-12. Wiley \& Sons, Chichester.

Welsh Government (2011) Delivering the independent mental health advocacy service in Wales. Available at: http://wales. gov.uk/docs/dhss/publications/111222advocacyen.pdf (accessed on 23/10/2013).

WHO (2003) Advocacy for Mental Health. World Health Organization, Geneva.

Yin R. (2008) Case Study Research: Design and Methods, Sage, London, UK. 